\title{
INTEGRATION OF PRANDTL'S EQUATION WITH THE AID OF QUADRATURE FORMULAE OF GAUSS TYPE
}

By

\author{
LAZAR DRAGOS
}

\author{
University of Bucharest, Bucharest, Romania
}

Abstract. The subject of this note is to present a method that can be used to integrate Prandtl's equation by means of Gauss-type quadrature formulae.

1. Introduction. There are two papers $[1,2]$, that treat the integration of Prandtl's equation using Gauss-type formulae. In both of these papers quadrature formulae for integrals of the form

$$
\int_{-1}^{+1} \frac{C^{\prime}(\eta)}{\eta-y} d \eta
$$

are constructed; however, the weight $w(x)=\left(1-x^{2}\right)^{-1 / 2}$ used by Ioakimidis is of no interest in aerodynamics. While the first author builds a Nyström-type numerical scheme for the solution of Prandtl's integro-differential equation, the second author does not even consider the solution of this equation. We may also mention [3], in which a general equation is considered.

In the present note, writing the integral (1) as an integral with a strong singularity and using a quadrature formula given by Monegato in [4], we reduce the integration problem of Prandtl's equation to a linear algebraic system that can easily be programmed for use in a computer. The test performed for the case of the elliptic wing shows that the solution obtained in this way is a very good approximation of the exact solution.

2. A new form of Prandtl's equation. We shall use Prandtl's equation for the compressible case [5] in the form

$$
\beta C(y)=\frac{a(y)}{2} \int_{-b}^{+b} \frac{C^{\prime}(\eta)}{\eta-y} d \eta+j(y)
$$

where, as usual, $C(y)$ is the circulation, $\beta=\left(1-M^{2}\right)^{1 / 2}, M$ being the Mach number in undisturbed motion and

$$
a(y)=\frac{x_{+}(y)-x_{-}(y)}{2}, \quad j(y)=-2 \int_{x_{-}(y)}^{x_{+}(y)}\left[\frac{x-x_{-}(y)}{x_{+}(y)-x}\right]^{1 / 2} \frac{\partial}{\partial x} h(x, y) d x .
$$

Received December 10, 1990.

1991 Mathematics Subject Classification. Primary 76G25. 
We denote by $x=x_{-}(y)$ and $x=x_{+}(y)$ the equations of the leading and trailing edge respectively, in the $(x, y)$-plane and by $z=h(x, y)$ the equation of the lifting surface. From the known conditions it follows that

$$
C( \pm b)=0 \text {. }
$$

In (2) the "prime" stands for the principal value in the Cauchy sense. From the definition of this value we have

$$
\int_{-b}^{+b} \frac{C^{\prime}(\eta)}{\eta-y} d \eta=\lim _{\varepsilon \rightarrow 0}\left(\int_{-b}^{y-\varepsilon}+\int_{y+\varepsilon}^{b}\right) \frac{C^{\prime}(\eta)}{\eta-y} d \eta .
$$

In the right-hand side, using the identity

$$
\frac{C^{\prime}(\eta)}{\eta-y}=\frac{\partial}{\partial \eta} \frac{C(\eta)}{\eta-y}+\frac{C(\eta)}{(\eta-y)^{2}}
$$

we may perform an integration by parts. Using (4) and remarking that

$$
C(y-\varepsilon)+C(y+\varepsilon)=2 C(y)+\varepsilon^{2} C^{4}(y)+\cdots,
$$

we deduce

$$
\begin{aligned}
\int_{-b}^{+b} \frac{C^{\prime}(\eta)}{\eta-y} d \eta & =\lim _{\varepsilon \rightarrow 0}\left[\left(\int_{-b}^{y-\varepsilon}+\int_{y+\varepsilon}^{b}\right) \frac{C(\eta)}{(\eta-y)^{2}} d \eta-\frac{2 C(y)}{\varepsilon}\right] \\
& =\int_{-b}^{+\infty} \frac{C(\eta)}{(\eta-y)^{2}} d \eta,
\end{aligned}
$$

the sign "*" standing for the "finite part" $[6,7]$.

Consequently, Prandtl's integro-differential equation is written as an integral equation in the form

$$
\beta C(y)=\frac{a(y)}{2} \int_{-b}^{+b} \frac{C(\eta)}{(\eta-y)^{2}} d \eta+j(y) .
$$

Making the change of variables

$$
y=b s, \quad \eta=b x
$$

and keeping the notation $C(s), a(s), j(s)$ for $C(b s), a(b s)$, and $j(b s)$, respectively, Eq. (8) becomes

$$
\beta C(s)=\frac{a(s)}{2 b} \int_{-1}^{+1} \frac{C(x)}{(x-s)^{2}} d x+j(s) .
$$

Conditions (4) imply for $C(s)$ the equations

$$
C( \pm 1)=0 \text {. }
$$

Hence, we shall look for the solution $C(s)$ in the form

$$
C(s)=\left(1-s^{2}\right)^{1 / 2} \mathrm{c}(s) .
$$

It is known [8] that the solution of the singular integro-differential equation (2) depends on the conditions imposed at the ends and that the conditions of the form $\left(4^{\prime}\right)$ imply solutions of the form (11). 
3. Quadrature formula. In [4], in Eqs. (4.1) and (4.2), Monegato gives the following quadrature formula of Gauss type:

$$
\int_{-1}^{+1} w(x) \frac{f(x)}{(x-s)^{2}} d x=\sum_{i=1}^{n} w_{i}^{\prime}(s) f\left(x_{i}\right)+R_{n}(f),
$$

where

$$
w_{i}^{\prime}(s)=\frac{q_{n}\left(x_{i}\right)-q_{n}(s)-q_{n}^{\prime}(s)\left(x_{i}-s\right)}{p_{n}^{\prime}\left(x_{i}\right)\left(x_{i}-s\right)^{2}},
$$

$x_{i}$ being the zero of the orthogonal polynomial $p_{n}(x)$ associated with the weight $w(x)$ and

$$
q_{n}(s)=\int_{-1}^{+1} w(x) \frac{p_{n}(x)}{x-s} d x .
$$

Formula (12) is valid for weights of the form

$$
w(x)=(1-x)^{\alpha}(1-x)^{\beta}, \quad \alpha, \beta>-1, \quad-1<s<1,
$$

when the orthogonal polynomials are the Jacobi polynomials. The formulae (12) are exact, i.e., $R_{n}(f)=0$ whenever $f(x)$ is a polynomial of degree $n-1$.

Isolating the term for which $i=k$ in the sum in (12) and passing to the limit for $s \rightarrow x_{k}$, we obtain the formula

$$
\int_{-1}^{+1} \frac{w(x) f(x)}{\left(x-x_{k}\right)^{2}} d x=\sum_{i=1}^{n} w_{i}^{\prime}\left(x_{k}\right) f\left(x_{i}\right)+w_{k}^{\prime}\left(x_{k}\right) f\left(x_{k}\right)+R_{n}(f),
$$

where the sign $\sum^{\prime}$ stands for $\sum$ with the case $i=k$ omitted, $w_{i}^{\prime}\left(x_{k}\right)$ is obtained from (13), and $w_{k}^{\prime}\left(x_{k}\right)$ is deduced by using l'Hôspital's rule in the form

$$
w_{k}^{\prime}\left(x_{k}\right)=\frac{q_{n}^{\prime \prime}\left(x_{k}\right)}{2 p_{n}^{\prime}\left(x_{k}\right)} \text {. }
$$

In the following, we shall use the formula (16) for $\alpha=\beta=1 / 2$.

Let us remark that a constant factor in the expression of the orthogonal polynomial $p_{n}$ disappears from the expressions of the coefficients $w_{i}^{\prime}(s)$. Hence we shall use the Jacobi polynomials without constant factors. For the weight function $w(x)=$ $\left(1-x^{2}\right)^{1 / 2}$ the Jacobi polynomials reduce to the Chebyshev polynomials of the second kind:

$$
p_{n}(x)=\frac{\sin (n+1) \theta}{\sin \theta}, \quad x=\cos \theta .
$$

It follows that

$$
x_{i}=\cos \frac{i \pi}{n+1}, \quad i=1, \ldots, n .
$$

Using Glauert's integral

$$
\frac{1}{\pi} \int_{0}^{\pi} \frac{\cos n \theta}{\cos \theta-\cos \sigma} d \theta=\frac{\sin n \sigma}{\sin \theta}, \quad n=0,1, \ldots,
$$

from definition (14) we deduce

$$
q_{n}(s)=-\pi \cos (n+1) \sigma, \quad s=\cos \sigma .
$$


Elementary calculations give

$$
q_{n}\left(x_{i}\right)=-(-1)^{i}, \quad q_{n}^{\prime}\left(x_{k}\right)=0, \quad p_{n}^{\prime}\left(x_{i}\right)=-\frac{(n+1)(-1)^{i}}{1-x_{i}^{2}},
$$

such that we may establish the formula

$$
\begin{aligned}
\frac{1}{\pi} \int_{-1}^{+1}\left(1-x^{2}\right)^{1 / 2} \frac{f(x)}{\left(x-x_{k}\right)^{2}} d x= & \frac{1}{n+1} \sum_{i=1}^{n}\left[1-(-1)^{i+k}\right] \frac{1-x_{i}^{2}}{\left(x_{i}-x_{k}\right)^{2}} f\left(x_{i}\right) \\
& -\frac{n+1}{2} f\left(x_{k}\right)+R_{n},
\end{aligned}
$$

the values of $x_{i}$ being given by (19).

4. Integration of Prandtl's equation. For the solution of Prandtl's equation (10) having the form (11), using formula (20) for $s=x_{k}$, and neglecting the term $R_{n}$, the following algebraic system is obtained:

$$
\left[\beta\left(1-x_{k}^{2}\right)^{1 / 2}+\frac{\pi}{b} \frac{n+1}{4} a_{k}\right] c_{k}+\sum_{i=1}^{n} A_{k i} c_{i}=j_{k}, \quad k=1, \ldots, n,
$$

where

$$
\begin{gathered}
a_{k}=a\left(x_{k}\right), \quad c_{k}=c\left(x_{k}\right), \quad j_{k}=j\left(x_{k}\right), \\
A_{k i}=\frac{\pi a_{k}}{2 b(n+1)}\left[1-(-1)^{i+k}\right] \frac{x_{i}^{2}-1}{\left(x_{i}-x_{k}\right)^{2}}, \quad i \neq k .
\end{gathered}
$$

Using also the notation

$$
A_{k k}=\beta\left(1-x_{k}^{2}\right)^{1 / 2}+\frac{\pi}{b} \frac{n+1}{4} a_{k}
$$

for system (21) we get the following form:

$$
\sum_{i=1}^{n} A_{k i} c_{i}=j_{k}, \quad k=1, \ldots, n,
$$

where $c_{1}, \ldots, c_{n}$ are unknown.

5. Coefficients of lift and moment. Coefficients of lift and moment are calculated with the formulae

$$
\begin{array}{ll}
C_{L}=\frac{2}{A} \int_{-b}^{+b} C(y) d y, & C_{D}=-\frac{2}{A} \int_{-b}^{+b} u(y) C(y) d y \\
C_{x}=\frac{2}{m A} \int_{-b}^{+b} y C(y) d y, & C_{z}=\frac{2}{m A} \int_{-b}^{+b} y u(y) C(y) d y
\end{array}
$$

where $A$ is the area of the wing in the $(x, y)$-plane, $m$ is the mean chord, and

$$
u(y)=\frac{1}{4 \pi} \int_{-b}^{+b} \frac{C^{\prime}(\eta)}{\eta-y} d \eta=\frac{\beta C(y)-j(y)}{2 \pi a(y)} .
$$


Performing the change of variables (9) we deduce

$$
\begin{aligned}
C_{L} & =\frac{2 b}{A} \int_{-1}^{+1} C(s) d s, & C_{D} & =-\frac{2 b}{A} \int_{-1}^{+1} u(s) C(s) d s, \\
C_{x} & =\frac{2 b^{2}}{m A} \int_{-1}^{+1} s C(s) d s, & C_{z} & =\frac{2 b^{2}}{m A} \int_{-1}^{+1} s u(s) C(s) d s,
\end{aligned}
$$

and using the quadrature formula

$$
\int_{-1}^{+1}\left(1-x^{2}\right)^{1 / 2} f(x) d x=\frac{\pi}{n+1} \sum_{i=1}^{n}\left(1-x_{i}^{2}\right) f\left(x_{i}\right)
$$

with $x_{i}$ given by (19), the following formulae follow:

$$
\begin{aligned}
& C_{L}=\frac{2 \pi b}{(n+1) A} \sum_{i=1}^{n}\left(1-x_{i}^{2}\right) c_{i}, \quad C_{D}=-\frac{2 \pi b}{(n+1) A} \sum_{i=1}^{n}\left(1-x_{i}^{2}\right) u_{i} c_{i}, \\
& C_{x}=\frac{2 \pi b^{2}}{(n+1) m A} \sum_{i=1}^{n}\left(1-x_{i}^{2}\right) x_{i} c_{i}, \quad C_{z}=\frac{2 \pi b^{2}}{(n+1) m A} \sum_{i=1}^{n}\left(1-x_{i}^{2}\right) x_{i} u_{i} c_{i},
\end{aligned}
$$

where

$$
u_{i}=\frac{\beta\left(1-x_{i}^{2}\right)^{1 / 2} c_{i}-j_{i}}{2 \pi a_{i}} .
$$

The sums in (28) are performed using a computer.

\section{Applications.}

Elliptic wing. In order to test the solution we use an elliptic wing for which we know the exact solution. Using the half-diameter along the direction of the free flow as characteristic length, the equation of the edge is written as $x^{2}+\left(y^{2} / b^{2}\right)=1$. It follows that

$$
x_{ \pm}(y)= \pm\left(1-y^{2} / b^{2}\right)^{1 / 2}, \quad h(x, y)=-\varepsilon x .
$$

From (3) and (9) we deduce

$$
a(s)=\left(1-s^{2}\right)^{1 / 2}, \quad j(s)=2 \pi \varepsilon a(s)
$$

For this problem the exact solution is

$$
C(y)=\kappa\left(1-y^{2} / b^{2}\right)^{1 / 2},
$$

where

$$
\left(\beta+\frac{\pi}{2 b}\right) \kappa=2 \pi \varepsilon
$$

With notation (11) we deduce $c(s)=\kappa$. Now putting

$$
A_{k i}=\left(1-x_{k}^{2}\right)^{1 / 2} A_{k i}^{\prime}
$$

it follows that the system

$$
\sum_{i=1}^{n} A_{k i}^{\prime} c_{i}^{\prime}=\beta+\pi /(2 b)
$$


must have the solution $c_{1}^{\prime}=c_{2}^{\prime}=\cdots=c_{n}^{\prime}=1$. For $b=10$ we obtain $c_{1}^{\prime}=c_{2}^{\prime}=$ $\cdots=c_{n}^{\prime}=1.000$; this fact shows that the proposed method is very good.

Rectangular wing. In this case the exact solution is not known. Considering $x_{ \pm}=$ \pm 1 it follows that $a(y)=1$ and $j=2 \pi \varepsilon$. Putting $c_{i}=2 \pi \varepsilon c^{\prime \prime}$ system (24) reduces to

$$
\sum_{i=1}^{n} A_{k i} c_{i}^{\prime \prime}=1, \quad k=1, \ldots, n,
$$

the coefficients $A_{k i}$ being yielded by (22) and (23) where $a_{k}=1$.

Calculating

$$
\begin{aligned}
k_{1}=\frac{1}{n+1} \sum_{i=1}^{n}\left(1-x_{i}^{2}\right) c_{i}^{\prime \prime}, & k_{2}=\frac{\beta}{n+1} \sum_{i=1}^{n}\left(1-x_{i}^{2}\right)^{3 / 2}\left(c_{i}^{\prime \prime}\right)^{2}, \\
k_{3}=\frac{1}{n+1} \sum_{i=1}^{n}\left(1-x_{i}^{2}\right) x_{i} c_{i}^{\prime \prime}, & k_{4}=\frac{\beta}{n+1} \sum_{i=1}^{n}\left(1-x_{i}^{2}\right)^{3 / 2} x_{i}\left(c_{i}^{\prime \prime}\right)^{2},
\end{aligned}
$$

we obtain

$$
\begin{array}{rlrl}
C_{L} & =\pi^{2} \varepsilon k_{1}, & & C_{D}=\pi^{2} \varepsilon^{2}\left(k_{1}-k_{2}\right), \\
2 C_{x} & =\pi^{2} b \varepsilon k_{3}, & 2 C_{z}=\pi^{2} b \varepsilon^{2}\left(k_{4}-k_{3}\right) .
\end{array}
$$

For $b=10$ the following values are obtained:

\begin{tabular}{|l|c|c|c|c|}
\hline & $k_{1}$ & $k_{2}$ & $k_{3}$ & $k_{4}$ \\
\hline$\beta=1$ & 0.2347 & 0.0907 & 0 & 0 \\
\hline$\beta=0.8$ & 0.2544 & 0.0856 & 0 & 0 \\
\hline
\end{tabular}

The result $C_{x}=C_{z}=0$ is natural due to the symmetry of the wing. The compressibility increases the lift and the drag. This is also a natural result. The value $2.307 \varepsilon$ obtained here for $C_{L}$ in the case of the incompressible fluid is below the value obtained by using Glauert's method [9] $(7.29 \varepsilon, 5.28 \varepsilon, \ldots)$, but the values given by Glauert are approaching the value given here if $\lambda(=2 \mathrm{~b} / \mathrm{m})$ increases, hence if the span is large with respect to the chord. This is just the approximation of the lifting line.

Consequently, the integration of Prandtl's equation with the aid of the quadrature formulae proves to be not only easy to perform on a computer but also very efficient.

\section{REFERENCES}

[1] N. I. Ioakimidis, A natural interpolation formula for Prandtl's singular integrodifferential equation, Int. J. Numer. Methods Fluids 4, 283 (1984)

[2] G. Monegato and V. Pennacchietti, Quadrature rules for Prandtl's integral equation, Computing 37, 31 (1986) 
[3] N. I. Ioakimidis and P. S. Theocaris, On the numerical solution of singular integrodifferential equations, Quart. Appl. Math. 37, 325 (1979)

[4] G. Monegato, On the weights of certain quadratures for the numerical evaluation of Cauchy principal value integrals and their derivatives, Numer. Math. 50, 273 (1987)

[5] L. Dragos, Method of fundamental solutions. A novel theory of lifting surface in a subsonic flow, Arch. Mech. 35, 575 (1983)

[6] K. W. Mangler, Improper integrals in theoretical aerodynamics, Br. Aero. Res. Council, R\&M 2424 (1951)

[7] Ch. Fox, A generalization of the Cauchy principal value, Canadian J. Math. 9, 110 (1957)

[8] N. I. Muskhelishvili, Singular Integral Equations, P. Noordhoff, Groningen, 1953

[9] Th. von Karman and J. M. Burgers, General aerodynamic theory-perfect fluids, in Aerodynamic Theory (Ed. W. F. Durand) vol. II, Springer, Berlin, 1935 